\title{
COMPORTAMENTO DO PESSEGUEIRO (Prunus persica L. BATSCH) CV. CHIMARRITA EM DIFERENTES SISTEMAS DE CONDUÇÃO'
}

\author{
CLEVISON LUIZ GIACOBBO ${ }^{2}$, JOÃO LUIZ CARVALHO FARIA ${ }^{3}$, OBERDAN DE CONTO ${ }^{4}$, ROBERTO FOSSA DE \\ BARCELLOS ${ }^{4}$, FERNANDO ROGÉRIO COSTA GOMES ${ }^{2}$
}

\begin{abstract}
RESUMO - A persicultura, no Brasil, é praticada há bastante tempo, porém poucos estudos foram realizados visando alternativas de manejo nos pomares para aumento de produtividade. Este trabalho teve por objetivo avaliar o desenvolvimento anual do diâmetro do tronco e produção nos terceiro e quarto anos, em pessegueiro cv. Chimarrita, enxertado sobre o porta-enxerto Capdeboscq. Foram avaliados quatro sistemas de condução: I- espaçamento de 0,5 m entre plantas, conduzido em 'Áxis colunar'; II e III - 1,0 m e 2,0 m, respectivamente, conduzidos em 'Y' e IV - 4,0 m em 'Vaso' (sistema tradicional). Entre as linhas de plantio o espaçamento foi constante de 5,0 m para todos os sistemas de condução. O pomar foi implantado em julho de 1997 na Fazenda experimental do Centro Agropecuário da Palma, propriedade da Universidade Federal de Pelotas, localizada no município de Capão do Leão, RS. Avaliou-se a taxa de crescimento anual do diâmetro do tronco, produtividade, qualidade físico-química das frutas e ocorrência de podridão parda. Os resultados obtidos na taxa de crescimento foram semelhantes em ambos os anos, porém observou-se maior incremento relativo no período 2000/2001, no sistema de condução I. A produtividade foi superior no sistema I e II diferindo do sistema IV, no entanto, a qualidade físicoquímica e a ocorrência de podridão parda foram semelhantes em todos os sistemas.
\end{abstract}

Termos para indexação: Pessegueiro; sistema de condução; densidade de plantio; aspectos produtivos.

\section{BEHAVIOR OF THE PEACH TREE (PRUNUS PERSICA L. BATSCH) CV. CHIMARRITA IN DIFFERENT TRAINING SYSTEMS}

\begin{abstract}
The peach culture has been practiced for a long time in Brazil but a few studies were performed aiming to define choices of orchard management for increasing productivity. The purpose of this study was to evaluate fruit production and annual growth rate of the trunk diameter in the third and fourth year after planting of peach-trees cv. "Chimarrita" on Capdeboscq rootstock. Four training systems were evaluated, as follow: I -spacing of 0,5 m among plants trained as "columnar axis"; II and III - spacing of 1 and $2 \mathrm{~m}$, respectively trained as an "Y shape" and IV - 4,0 $\mathrm{m}$ as an "open vase" (traditional system). The space between tree rows was steady in 5,0 m for all the training systems. The orchard was implanted in July 1997 in the Experimental Farm of Centro Agropecuário da Palma, Universidade Federal de Pelotas located in a rural district of Capão do Leão, RS. The annual growth rate of the trunk diameter, productivity, physicochemical quality of fruits and rottenness occurrence were evaluated. The trunk rate was similar in both years, but a greater relative increasing was observed in 2000/2001 period for training system I. The productivity was higher in system I and II differing from the IV system, nonetheless the physicochemical quality and rottenness occurrence were similar in all systems.
\end{abstract}

Index Terms: Peach tree; training systems; planting density; productive aspect.

\section{INTRODUÇÃO}

Até a década de 50, somente $20 \%$ do pêssego consumido no Brasil foi proveniente da produção nacional, enquanto o restante era importado fresco ou industrializado. Com medidas restritivas à importação, conseguiu-se que a cultura obtivesse desenvolvimento significativo e alcançasse maior expansão. Na região sul, em particular no Rio Grande do Sul, atingiu maior importância a partir da década de 60, quando programas de melhoramento genético deram grande estímulo à cultura (Sachs \& Campos, 1998).

Visando incrementar lucros com esta cultura, busca-se aumentar a produtividade e antecipar a entrada de produção sem perder em qualidade no produto final, desta forma, o estudo de novas formas de condução e densidade de plantio se faz necessário. Para Fachinello (1999), a tendência da fruticultura moderna, em algumas culturas, é o cultivo em sistemas de pomares adensados, com uso de mudas pré-formadas, que rapidamente proporcionam a cobertura do solo, melhorando a capacidade de interceptação da luz solar, combinando e otimizando todos os fatores de produção.

A técnica de plantio em alta densidade surgiu no início dos anos 70 na Itália, com o objetivo de reduzir os custos das operações de poda, raleio e colheita, visando ainda antecipar a entrada em produção das plantas e acelerar a obtenção da produtividade máxima dos pomares (Guerriero \& Loreti, 1978; Guerriero et al., 1980; Loreti, 2001).

Para Salaya (1999), vários são os sistemas e derivações empre- gados na formação e condução de plantas frutíferas em função de sua fisiologia e necessidades práticas, ou de ambas, sendo atualmente os mais utilizados as formas em volume, com a copa se desenvolvendo em todos os sentidos, com eixo central (livre ou globoso, pirâmides ou cone, fuso, fusito e minifusito), sem eixo central (vaso, vasito e Y transversal livre) e com eixo a meia altura (eixo modificado e vaso retardado).

Em pomares de frutíferas de caroço conduzidos em baixa densidade (menos de 800 plantas.ha $^{-1}$ ) é necessário formar as plantas com copas grandes para melhor aproveitamento da área, podendo ser utilizado os sistemas em forma de 'Vaso', 'Vaso Retardado' e 'Guia Modificado'. Em plantios de média densidade (800 a 2.500 plantas.ha' ${ }^{-1}$ ), as formas de condução mais utilizadas são 'Palmeta Livre', 'Y Transversal' e 'V', enquanto que no sistema de alta densidade (mais de 2.500 plantas.ha ${ }^{-1}$ ), as mais utilizadas são 'Fuso', 'Y Transversal' ou 'Tatura Trellis' e 'Áxis Colunar' (Fideghelli, 1994; Fachinello, 1999; Salaya, 1999).

Com o propósito de avaliar o desenvolvimento anual do diâmetro do tronco e a produção em pomar de pessegueiro da cultivar 'Chimarrita', enxertada sobre o porta-enxerto 'Capdeboscq' em diferentes sistemas de condução e densidade de plantio, procedeu-se o acompanhamento nos terceiro e quarto anos de produção.

\section{MATERIALEMÉTODOS}

Os trabalhos foram conduzidos a campo, no período de março de 2000 a dezembro de 2001, na Faculdade de Agronomia Eliseu Maciel

\footnotetext{
${ }^{1}$ (Trabalho 125/2002). Recebido: 13/08/2002. Aceito para publicação: 14/05/2003. Trabalho realizado como parte de dissertação de Mestrado do primeiro autor.

${ }^{2}$ Aluno do PPGA, área de concentração em Fruticultura de Clima Temperado. FAEM/UFPel. E-mail: giacobbo@ ufpel.tche.br, Fone: (053) 275-7124. R. Argolo 1418/ 102, 96015-160. Pelotas, RS. Autor para correspondência.

${ }^{3}$ Professor DR, do Departamento de Fitotecnia da Faculdade de Agronomia Eliseu Maciel, Universidade Federal de Pelotas. Fone: (053)275-7326. Cx. P. 354, 96010900 - Campus Universitário E-mail: jfaria@ufpel.tche.br.

${ }^{4}$ Graduando em Agronomia, Faculdade de Agronomia Eliseu Maciel, Universidade Federal de Pelotas. Fone: (053)275-7124. Cx. P. 354, 96010-900 - Campus Universitário.
} 
(FAEM) - Fazenda Agropecuária da Palma, propriedade da Universidade Federal de Pelotas - UFPel, localizada no município de Capão do Leão/ RS e no laboratório de Fisiologia de pós-colheita da Universidade.

O experimento constituiu-se de um pomar de pessegueiro, cultivar 'Chimarrita', enxertado sobre o porta-enxerto 'Capdebosq', implantado em julho de 1997. O plantio foi disposto em quatro blocos e cada bloco dividido em quatro parcelas, variando diferentes densidades de plantas, em função dos tratamentos. A distância entre filas foi mantida constante em 5 metros. As filas foram dispostas na orientação Norte-Sul.

Os tratamentos foram definidos como: a) Sistema de condução I: conduzido em forma de 'Áxis colunar', com espaçamento de $5 \times 0,5 \mathrm{~m}$, 25 plantas/parcela (4000 plantas.ha ${ }^{-1}$ ); b) Sistema de condução II: conduzido em "Y", com espaçamento de 5 x 1 m, 13 plantas/parcela (2000 plantas.ha-1); c) Sistema de condução III: conduzido em "Y", com espaçamento de $5 \times 2 \mathrm{~m}, 7$ plantas/parcela (1000 plantas.ha $\left.^{-1}\right)$ e d) Sistema de condução IV: conduzido em 'Vaso', com espaçamento de $5 \times 4$ m, 4 plantas/parcela (500 plantas.ha $\left.^{-1}\right)$.

O delineamento experimental foi em blocos casualizados, com quatro repetições. Cada bloco foi constituído por três linhas de plantio, onde as linhas externas foram consideradas bordaduras e a central linha útil, contendo 4 parcelas em cada bloco, com os diferentes tratamentos, sendo as plantas das extremidades de cada parcela consideradas bordaduras.

As variáveis analisadas foram: a) Taxa de crescimento anual do diâmetro do caule, determinada pela mensuração anual do diâmetro do tronco medido a $10 \mathrm{~cm}$ acima do ponto da enxertia. As medidas foram realizadas nos meses de novembro dos anos de 1999, 2000 e 2001, em duas direções, paralela e perpendicular à linha de plantio, utilizou-se paquímetro digital e realizou-se a média das medições. Através desta, foi calculado a taxa de crescimento anual do tronco pela fórmula:

$\mathrm{Tc}=\left(\mathrm{X}^{2}-\mathrm{X}^{1}\right) / \mathrm{X}^{1 * 100}$, onde: Tc (Taxa de crescimento em percentagem); $\mathrm{X}^{1}$ (Valor da medida do ciclo anterior); $\mathrm{X}^{2}$ (Valor da última medida);

b) Dados de produção: foram realizadas colheitas nos dias 14/12 e 17/12 de 2000 e 23/11 e 28/11 de 2001, quando as frutas atingiram o ponto de maturação. No momento da colheita foram avaliados os seguintes parâmetros: 1) Produtividade estimada: a produção total, obtida em Kg na parcela foi estimada para um hectare; 2) Tamanho da fruta (altura e diâmetro): determinado através da amostragem de 20 frutos/parcela e medidos com um paquímetro digital; 3) Peso médio das frutas: determinado através de pesagem em balança mecânica com capacidade para $25 \mathrm{~kg}$ e realizado à média para cada parcela; 4) Firmeza da polpa $(\mathrm{N})$ : realizada com penetrômetro manual através de duas leituras na região equatorial das frutas em lados diametralmente opostos e após e remoção da epiderme; 5) Determinação do $\mathrm{pH}$ : realizado diretamente no suco das frutas amostradas, com o uso de um medidor de $\mathrm{pH}$ da marca Analion e expressos em medidas de $\mathrm{pH}$; 6) ATT - Acidez total titulável $\left(\mathrm{Cmol} . \mathrm{L}^{-1}\right)$ : determinada a partir de $10 \mathrm{~mL}$ de suco de pêssego, diluídos em $90 \mathrm{ml}$ de água destilada. A solução foi titulada com $\mathrm{NaOH} 0,1 \mathrm{~N}$ até a mudança de coloração, usando o indicador fenolftaleina; 7) SST - Sólidos Solúveis Totais ( ${ }^{\circ}$ Brix): leitura direta em refratômetro manual, marca Atago N1; 8) Ocorrência de podridão parda [Monilinia fruticola (Wint) Honey]: no momento da colheita foram recolhidas as frutas podres e contadas para verificar a incidência de podridão parda, expressa em porcentagem. Em ambos os anos não foram efetuados aplicações de fungicidas para o controle de podridão.

As práticas de manejo, principalmente podas, foram realizadas na mesma data em todos os tratamentos, seguindo as indicações técnicas para a cultura e considerando os diferentes sistemas de condução das plantas.

\section{RESULTADOS}

Com relação à taxa de crescimento, observou-se que as plantas apresentaram comportamento semelhante na porcentagem de crescimento do tronco, não apresentando diferenças nos anos de 2000 e 2001 entre os diferentes sistemas de condução. Entretanto, quando considerado o valor absoluto do diâmetro do tronco, as plantas conduzidas em forma de vaso, sistema 'IV', apresentaram desenvolvimento do tronco superior ao sistema de condução em forma de áxis colunar - 'I' e sistema em Y - 'II', (Tabela 1).

Quanto à produtividade obtida nos anos de 2000 e 2001 (terceiro e quarto ano de produção), verificou-se diferenças significativas entre os sistemas de condução utilizados. O sistema II, conduzido em forma de Y, com 2000 plantas/ha, no ano de 2000, apresentou maior produtividade $(6,57 \mathrm{t} / \mathrm{ha})$ em relação ao sistema IV, conduzido em forma de vaso, com 500 plantas/ha (2,93 t/ha). No ano de 2001, o sistema II, conduzido em forma de Y, com 2000 plantas/ha e produtividade de 13,19t/ha, foi superior aos sistemas III e IV, conduzidos em forma de Y e vaso, com 1000 e 500 plantas/ha e com produtividade de 7,12 e 5,38 t/ha, respectivamente. Observou-se ainda que o sistema IV, conduzido em forma de vaso, também foi inferior ao sistema de condução I, conduzido em forma de áxis colunar, com 4000 plantas/ha, cuja produtividade foi de 10,97 t/ha, conforme tabela 1. Quando considerado os dois anos em conjunto, os sistemas I e II, conduzidos em forma de áxis colunar e Y, respectivamente, apresentaram rendimentos superiores ao sistema tradicional IV, em forma de vaso (Tabela 1)

A análise físico-química das frutas não apresentou diferenças significativas em todas variáveis analisadas. Nos anos 2000 e 2001, não houve influência do sistema de condução, onde os valores médios relativos foram: ATT - acidez total titulável $\left(0,32\right.$ e 0,28 cmol.L $\left.L^{-1}\right), \mathrm{pH}(4,12$ e 4,00), SST -sólidos solúveis totais $\left(12,21\right.$ e $\left.13,17^{\circ} \mathrm{Brix}\right)$, firmeza da polpa $(60,47$ e $55,38 \mathrm{~N})$, altura $(59,35$ e $55,39 \mathrm{~mm})$ e diâmetro de frutos $(62,77$ e $55,57 \mathrm{~mm})$.

No ano de 2000, em decorrência das condições climáticas terem sido favoráveis para o não desenvolvimento de podridões, não foram verificadas frutas com podridão parda [Monilinia fruticola (Wint) Honey]. Em 2001, a ocorrência de podridão parda foi maior, mas não observou-se influência quanto ao sistema de condução utilizado, apresentando porcentagem média relativa de $27,06 \%$ de frutos contaminados.

TABELA 1 - Efeito de quatro sistemas de cultivo sobre a taxa de crescimento do tronco, estimada a partir do diâmetro medido, 10cm acima do ponto de enxertia em pessegueiro cv 'Chimarrita', safras 2000 e 2001 (FAEM/UFPel, 2002)

\begin{tabular}{|c|c|c|c|c|c|c|c|}
\hline $\begin{array}{c}\text { Sistemas de cultivo/ } \\
\text { espaçamentos }\end{array}$ & \multicolumn{2}{|c|}{$\begin{array}{l}\text { Diâmetro do tronco } \\
\qquad(\mathrm{mm})\end{array}$} & \multicolumn{2}{|c|}{$\begin{array}{c}\text { Tx. de cresc. do } \\
\text { diâmetro do tronco }(\%)\end{array}$} & \multicolumn{2}{|c|}{$\begin{array}{l}\text { Produtividade estimada } \\
\qquad\left(\mathrm{t} . \mathrm{ha}^{-1}\right)\end{array}$} & \multirow{2}{*}{$\begin{array}{c}\begin{array}{c}\text { Somatória da } \\
\text { produtiv. } \\
\text { (t.há }^{-1} \text { ) }\end{array} \\
2000 / 01\end{array}$} \\
\hline I - Áxis Colunar $/ 0.5 \mathrm{~m}$ & $2000^{1}$ & $2001^{2}$ & $2000^{1}$ & $2001^{2}$ & 2000 & 2001 & \\
\hline $\mathrm{II}-\mathrm{Y} / 1 \mathrm{~m}$ & $45,88 \quad \mathrm{c}$ & $51,99 \quad \mathrm{c}$ & $4,47^{\mathrm{ns}}$ & $13,36^{\text {ns }}$ & $3,63 \mathrm{ab}$ & $10,97 \mathrm{ab}$ & $15,71 \mathrm{a}$ \\
\hline $\mathrm{III}-\mathrm{Y} / 2 \mathrm{~m}$ & $51,74 \mathrm{bc}$ & 60,74 bc & 6,59 & 17,42 & $6,57 \mathrm{a}$ & $13,19 \mathrm{a}$ & $19,71 \mathrm{a}$ \\
\hline IV - Vaso/4m & $57,87 \mathrm{ab}$ & $68,86 \mathrm{ab}$ & 7,20 & 19,15 & $4,78 \mathrm{ab}$ & $7,12 \mathrm{bc}$ & $12,90 \mathrm{ab}$ \\
\hline & $67,33 a$ & $76,22 \mathrm{a}$ & 7,80 & 13,51 & $2,93 \mathrm{~b}$ & $5,38 \quad \mathrm{c}$ & $8,31 \mathrm{~b}$ \\
\hline C. V. $(\%)$ & 9,72 & 6,75 & 32,56 & 45,88 & 28,35 & 26,08 & 36,38 \\
\hline
\end{tabular}

ns: não significativo.

Médias seguidas por letras distintas na coluna, diferem significativamente entre si pelo teste de Tukey em nível de 5\% de significância.

1: valores relativos ao período de novembro de 1999 a novembro de 2000.

2: valores relativos ao período de novembro de 2000 a novembro de 2001. 


\section{DISCUSSÃO}

No que se refere à taxa de crescimento de tronco, ainda que os dados obtidos não apresentem diferenças significativas, no terceiro e quarto ano de crescimento é possível verificar que o sistema de cultivo I apresentou, relativamente, maior incremento na variação da taxa de crescimento no período considerado (2000/2001), principalmente em relação ao sistema de cultivo IV. No sistema I, o incremento foi de $199 \%$ $(4,47 \%$ para $13,36 \%)$, enquanto no sistema IV foi de $73 \%$ (7,80\% para 13,51\%), (Tabela 1).

Em relação à taxa de crescimento de tronco, observou-se maior incremento relativo no sistema I, conduzido em forma de 'áxis colunar' do que no sistema IV, conduzido em forma de vaso. Isto sugere que o sistema IV estaria próximo à estabilidade de crescimento, enquanto o sistema I poderia ainda não ter atingido a estabilidade referida devido a maior densidade de plantio utilizada. É importante salientar que a forma em que as plantas foram conduzidas no sistema I, áxis colunar, é diferenciada dos demais sistemas e que a introdução da poda de verão, em 1999, pode ter ocasionado desequilíbrio, provocando estresse e conseqüentemente influenciando no incremento da taxa de crescimento. Day (1998) e Salaya (1999) relatam que o aumento na circunferência do tronco tem relação direta com a severidade da poda, principalmente quando realizada nas primeiras estações de crescimento, pois quanto mais severa for a poda menor é o desenvolvimento do tronco. Isto justifica o menor desenvolvimento inicial do sistema de cultivo I, que por ter maior densidade de plantas exige poda mais severa, principalmente para a retirada de ramos longos, objetivando aproximar a produção do eixo central.

A poda verde realizada no experimento, no mês de dezembro de 1999, mais intensa no sistema I, conduzido em forma de áxis colunar, visando adequar a arquitetura das plantas ao sistema, pode ter interferido na baixa taxa de crescimento no ano de 2000. Após o período de um ciclo vegetativo, as plantas provavelmente superaram o estresse provocado pela poda e retomaram seu crescimento.

Com relação à produtividade, dentre os sistemas de condução estudados, os sistemas I e II são mais produtivos em relação ao sistema IV o qual é o mais utilizado na região. Esta observação leva em consideração o somatório das safras 2000 e 2001, terceira e quarta safras, respectivamente. Resultados semelhantes também foram observados por Bargioni et al. (1985) e Recupero et al. (1985), os quais citam que populações próximas a 2000 plantas.ha $^{-1}$ são mais indicadas para obtenção de alta produtividade e melhor qualidade de frutas, além de facilitar os tratos culturais.

A baixa produtividade obtida em todos os sistemas na safra de 2000 foi resultante provavelmente, da queda antecipada das folhas. Como consequiência ocorreram brotações e floração antecipada em todo o pomar (maio de 2000) prejudicadas pela ocorrência de geadas no período de inverno.

É importante observar que até o quarto ano de cultivo, mesmo não havendo diferenças na produtividade entre os sistemas I, áxis colunar e II, Y, o custo de implantação dos pomares é diferenciado. Para a implantação do sistema II o custo com a aquisição das mudas é inferior, já que, metade do número de mudas se faz necessário. Este fato deve ser considerado no momento da decisão de qual sistema adotar para a implantação de novos pomares, porém, é importante salientar que esta avaliação só poderá ser conclusiva e suficientemente segura, considerando-se mais anos de estudos.

Pelas análises físico-químicas, nos dois anos considerados, observou-se que os diferentes sistemas não influenciaram estas variáveis, o que já havia sido observado em anos anteriores, mas isso não significa que não ocorram diferenças no futuro. Bargioni et al. (1985), estudando durante dez anos diferentes sistemas de condução em pessegueiros, relataram que mesmo nos sistemas que apresentaram maior produtividade, a qualidade e o diâmetro de frutas somente diferiram a partir do quinto ano de produção.

Foi verificado ainda, nas safras estudadas, que a variação na densidade de plantio, no sistema de poda e conseqüentemente na área e estruturação da copa, não proporcionaram efeitos sobre a ocorrência de doenças fúngicas, principalmente quanto à incidência de podridão parda [Monilinia fruticola (Wint) Honey]. Provavelmente fatores como luminosidade, circulação do ar e retenção de umidade não foram suficientemente significativos para provocar doenças. Desta forma, o maior adensamento, com copa mais compacta, não necessariamente resulta em maior vulnerabilidade ao ataque de agentes patogênicos, como o causador da podridão.

Este último aspecto somente será melhor esclarecido em estudos que se seguirão nos próximos anos. Entretanto, parece haver uma tendência no aumento de produção com o adensamento do pomar. Maior critério na quantificação destes ganhos deverá ser adotado, pois é necessário levar em consideração a compensação dos gastos maiores com a implantação e manutenção do pomar, assim como as condições futuras, já que existe indicação da ocorrência de uma aceleração no processo de envelhecimento de plantas quando cultivadas em alta densidade, comprometendo desta forma a longevidade do pomar com conseqüente influência na rentabilidade.

\section{CONCLUSÕES}

Baseado nas terceira e quarta safras colhidas em plantas de pessegueiro da cv. 'Chimarrita' sobre o porta-enxerto 'Capdeboscq', pôde-se concluir que os sistemas de condução estudados neste trabalho ('áxis colunar', 'Y' e 'vaso'):

1) não interferem na qualidade das frutas;

2) os sistemas 'áxis colunar' com 0,5 m e 'Y' com 1,0 m entre plantas proporcionam maior produtividade que o sistema em 'vaso';

3) os sistemas de condução não influenciam na incidência de podridão parda.

\section{REFERÊNCIASBIBLIOGRAFIAS}

BARGIONI, G.; LORETI, F.; PISAN, P. L. Ten years of research on peach and nectarine in a hight density system in the Verona area. Acta Horticulturae. Wageningen, v. 173, p. 229-309, 1985.

DAY, K. R. Portainjertos, densidad de plantación y sistemas de manejo del monte, para nectarines, durazneros y ciruelos. In: CURSO INTERNACIIOANALDE FRUTICULTURADE CLIMATEMPLADO-FRIO, 1998, Mendonza. Anais... Mendonza/Argentina: INTA(Instituto Nacional de Tecnologia Agropecuaria Centro Regional Cuyo), 1998, cap. 2, p. 1-8.

FACHINELLO, J. C. Avanços nos sistemas de condução e densidades de plantio em fruteiras de caroço. In: ENCONTRO NACIONAL SOBRE FRUTICULTURA DE CLIMA TEMPERADO, 2., 1999, Fraiburgo. Anais... Caçador: EPAGRI, 1999. p. 9-13.

FIDEGHELLI, C. La poda del duraznero In: CURSO INTERNACIONAL DE FRUTALES DE CAROZO, 1994, General Roca. Anais... General Roca, Argentina: INTA(Instituto Nacional de Tecnologia Agropecuária Centro Regional Cuyo), 1994. cap. 4.2, p. 4.2 a 4.2-15

LORETI, F. Formas atuais de condução e densidade de plantio do pessegueiro na Itália. In: ENCONTRO NACIONALSOBREFRUTICULTURADE CLIMA TEMPERADO, 4., 2001, Fraiburgo. Anais... Caçador: EPAGRI, 2001.p. 133-142.

GUERRIERO, R.; LORETI, F. Evoluzione delle forme di allevamento e di distanze di impianto nel pesco. L'Italia Agrícola, v. 10, p. 75-91, 1978.

GUERRIERO, R.; LORETI, F.; MORINI, S.; NATALI, S. Eight years of observation on a peach double-row planted orchard. Acta Horticulture, Wageningen, v. 114, p. 365-384, 1980.

RECUPERO, S.; MONASTRA, F.; DAMIANO, C. Study on planting densities of peach and nectarine cultivars on different rootstocks. Acta Horticulturae, Wageningen, v. 173, n. 3, p. 311-322, 1985.

SACHS, S.; CAMPOS, A. D. O pessegueiro, In: MEDEIROS, C. A.; RASEIRA, M. do C. B. (Ed.) A Cultura do pessegueiro. Brasília: EMBRAPA-SPI; Pelotas: EMBRAPA-cpact, 1998. p. 13-19.

SALAYA, G. F. G. Fruticultura: el potencial productivo. 2. ed. México: ALFAOMEGA, Ediciones Universidad Católica de Chile, 1999. 342p. 